\title{
LA SICILIA DESCRITA DELLA PENNA DE UN AUTORE DEL X SECOLO: IBN HAWOAL *
}

\author{
Por \\ ABDERRAHMÁN TLILI
}

\section{Configurazione della terra (Kitâb șurat al-arḍ: l'ordine della opera e il suo programma.}

Sembra che ci siano state diverse redazione successive dell'opera. Una prima edizione ha devuto portare lo stesso titolo di quella di al-Istakhri: Descrizione degli itinerari e degli stali. Ibn Hawqal ha proceduto a un costante aggiornamento del suo lavoro, e gli storici sono d'accordo nel fissare all'anno 988 la data della revisione definitiva. (1).

Il piano dell'Opera è copiato da quello di Istakhir; comincia con una introduzione generale sulla terra: "Fisionomia della terra". Vengono in seguito le descrizioni delle province, con la penisola arabica in testa: "ho cominciato dal territorio degli arabi, di cui ho fatto una regione distinta perchè essa contiene la Ka'ba e la Mecca, madre delle città, ed è ai miei occhi il centro di tutto l'insiemen, (2); viene in seguito la descrizione del mare di Persia che la circonda. Ci sono in tutto sedici provincie: I'Arabia, il Maghreb, I'Egitto, la Siria, I'Alta Mesopotania, I'Iraq, il Khuzistan, il Fars, il Kerman, il Sind, I'insieme ArmeniaAzerbaidjan, il Djibal, il Dailan-Tabaristan, il Seistan, il Khorassan, la Transexina; a queste bisogna aggiungere i quattro insiemi che costituiscono il mare di Persia, il mare Mediterraneo, il mar Caspio e la Steppa del Khorassan e del Fars.

( *Ill nostro studio è basato quasi interamente sull'opera di Ibn Hawqal Confi-

\footnotetext{
(*) m. dopo 366/997.

(1) G. Wiet, Introduction à la littératura arabe (Paris, 1966) p. XIII.

(2) Ibn Hawqal, Configuration de la terre, p. 6, 18.
} 
guration de la terra tradotto in francese da J. H. Kramers e G. Wiet. Per maggiori referenze, rinviamo i lettorilinteressati alla nostra bibliografia in fine articolo.

Il programma è presentato nell'introduzione:

«Ho tracciato per ciascuna delle parti alle quali ho consacrato un capitolo intero, un disegno precisando la posizione di questa regione, con la menzione delle località e delle regioni circostanti, delie città e delle province che contiene, i suoi tributi e le sue rendite, i suoi fiumi e isuoi mari. Vi ho riunito tutto ciò che si dovrebbe conoscere su ogni regione, vale a dire le diverse fonti di ricchezza, le imposizioni, le decime, le imposte fondiarie, le distanze negli itinerari, l'esportazione e gli articolo di commercion. (3).

Si tratta dunque d'un atlas dell, Islam, largamente commentato. In effetti è nella doppia ottica di un cartografo, ma di un cartografo del solo Islam che lbn Hawqal immaginò la sua opera geografica. Nonostante ciò i dati accumulati durante i suoi viaggi lo incitarono a concentrare la maggior parte dei suoi sforzi nel testo stesso.

II Kitâb surat al-ard contiene la descrizione del mondo musulmano con I'indicazione di ogni regione, dando priorità alle caratteristiche geografiche piuttosto che a quelle politiche.

Senza uscire dal quadro generale dell'Islam, né dalla presentazione di ciascuna provincia presa una ad una, Ibn Hawqal aggiunge alla sua descrizione delle osservazioni sui diversi paesi e popoli vicini dell'Islam che gli sembrarono di particolare interesse: Sudanesi, Nubiani, cristiani di Spagna, ecc.

\section{Il suo metodo: la sua preoccupazione di informare e la critica personale}

"Sin da giovane ho ardentemente desiderato conoscere la storia dei diversi paesi, ed informarmi sulle condizioni delle grandi città. Interrogavo senza tregua i viaggiatori che avevano percorso il mondo, i cortigiani e i mercanti e leggevo le opere concernenti questa materia. Quando mi capitò di incontrare un uomo che stimavo veritiero e che pensai ben informato sugli argomenti di cui lo avrei intrattenuto, gli feci ripetere l'avvenimento in cui avevo creduto e misurai la sua veridicità. Ritenni la sua narrazione, studiai i suoi itinerari, le sue descrizioni di paesi, e constatai che la maggior parte delle sue dichiarazioni erano errate e che il mio interlocutore ignorava quasi tutto di ciò che reccontava. Lo invitai allora a riprendere il racconto che gli avevo domandato e gli feci ascoltare la descrizione che avevo preso altrove. Confrontai così con lui i dire di un altro informatore che aveva visto ciò di cui si trattava. In seguito confrontai queste due versioni a una terza, con parzialità e senza partito preso: le testimonianze differivano ancora e le posizioni erano contraddittorie. Questo alla lunga, nom mi rese che ancor più avido di soddisfare al gusto

(3) Ibn Hawqal, op. cit., p. 2. 
che risentivo per il viaggio, senza contare l'attrazione per i pericoli e il desiderio di stimare esattamente la posizione delle città, le condizioni delle metropoli, i limiti delle province e delle regioni. Durante le mie peregrinazioni, né il libro di Ibn Khurdadhbeh, né quello di Djaihani, né il preciso Abul-Faradj Qudama ibn'far non mi abbandonarono. Le lettura delle prime due opere mi avevano assorbito tanto che devo chiedere perdono a Dio d'averle prese, e mi avevano distolto nella mia obbligazione di coltivare altre scienze utili e di praticare dei doveri imperativi». (4).

Questo testo ci è prezioso per stabiliere quale sia il metodo del nostro geografo. Esso è reppresentativo di quell'epoca.

1) I'informazione libresca resta una fonte essenziale; il prestito resta sempre in una certa misura, la sanzione della conoscienza, poiché Ibn Hawqal non cerca di rivoluzionare la tradizione ma di perfezionarla;

2) I'informazione orale viene ad aggiungersi a quella precedente, egli interroga i viaggiatori, i mercanti, i pellegrini ecc.

3) infine ricco delle sue nuove informazioni, dell'esperienza personale che niente può rimpiazzare, verifica e controlla le informazioni raccolte nei libri e nei racconti orali.

Così, Ibn Hawqal si riferisce ad altri autori, vecchi ed arabi. Nonostante ciò egli proclama la sua libertà nei confronti dei suoi predecessori e mette in risalto il suo spirito critico. Si sappia che «Quest'opera non potrebbe esser confrontata a quella di Djaihani, e non concorda nemmeno con i dati di Ibn Khurdadhbeh. E al lettore, e che Dio lo benedica, di considerare i fatti sui quali ha dei dubbi, di riflettere alla maniera di chi, scegliendo la verita, si libera di un gran numero di racconti d'informatori o di viaggiatori ignoranti e bugiardi che non aspirano alla verità» (5).

L'informazione personale sistematizzata è il fondamento del suo metodo, e l'osservazione personale sarà presa come criterio costante di riferimento. L'amore por la verità si traduce con una riserva per la tradizioni incontrollabili, così pure con assenza significativa di «mirabilia». Nonostante ciò dobbiano quì marcare un limite quanto all'oggettività del nostro viaggiatore, poichè Ibn Hawqal è parziale nei suoi scritti sulla Spagnia e la Sicilia, dove I'informazione, reccolta presso mercanti, non riflette la realtà. Ci troviano qui di fronte a un problema che riprenderemo più tardi.

\section{LA SICILIA}

\section{SITUAZIONE E ASPETTI GENERALI DELL'ISOLA}

Di tutte le isole del mediterraneo, "la più prospera, grazie all'Islam e la

(4) Ibn Hawqal, op. cit., p. 321, 322.

(5) Ibn Hawqal, op. cit., p. 5. 
sua popolazione, è la Sicilia: è la più grande di tutte, la meglio approvviggionata, la meglio difesa, dall'energia degli emigranti del Maghreb che vi affluiscono" (6).

Sottolineiamo subito l'importanza che rappresenta la possessione della Sicilia, e la presenza dei musulmani nel Mediterraneo per la loro sicurezza e la loro economia, poichè l'isola occupa una posizione strategica tra i due bacini del mediterraneo e conferisce ai suoi possessori il controllo della parte ovest del mare. L'isola fu conquistata sotto gli Aghlabiti del Maghreb, verso la matà del IX secolo. Pur senza essere diventato un lago mediterraneo, dopo la conquista della Sicilia, il Mediterraneo è passato sotto il controllo arabo e il commercio marittimo può effettuarsi senza ingombrare le rive della Spagna e del Maghreb fino a quelle dell'Eqitto e della Siria.

Ion Hawqal ci insegna che l'isola, soprattutto la città di Palermo «è una Marca contro l'Europa, una contrada che fa fronte al nemico: la guerra santa persiste continuamente e il richiamo alle armi vi restò costante da quando la Sicilia è stata conquistata" (7).

L'isola è costituita soprattutto da montagne, da castello e fortezze. Ci sono molti conventi militari situati in riva al mare. Il sovrano possiede una flotta.

"Tra i paesi che sono sotto il dominio musulmano, dal punto di vista economico si può collegare la Sicilia alla Spagnan (8). Gran parte del suo territorio è abitato e coltivato. Ibn Hawqal si trovò in questa regione nell'anno 362 (973) e afferma che al momento in cui egli vi era in visita, la situazione del paese era peggiorata passando dall'abbondanza alla miseria. In effetti vedremo che il quadro economico tracciato è abbastanza negativo.

Il geografo situa la Sicilia confrontandola alla Spagna, al Maghreb e all'impero bizantino, indicando la città di Ifriqiya che gli sono in faccia: "essa situata in pieno mare, all'est della Spagna e in faccia alla regione del Maghreb a partire da Ifriqiya, Badja e tabarqa fino a Marsa I-Kharaz. All'ovest, in mare si trova la Corsica. A sud c'è l'isola di Pantelleria. All'est, sul litorale del continente in cui si trova Costantinopoli c'è la città di Reggio, poi le province della Calabria" (9).

Per finire il testo ci informa sulla popolazione dell'isola, costituita come sappiamo da emigrati del Maghreb. Infatti la Sicilia dopo la Spagnia, fu un fronte dell'espanzione berbera: "quando un esercito berbero, proveniente dal Maghreb penetra nei paesi bizantini, esso li devasta e li deruba, (...) è così che i magrebini hanno imposto agli abitanti della Calabria un tributo annuo di parecchi denari che essi prelevavano su di loro" (10).

(6) Ibn Hawqal, op. cit., p. 197, 198.

(7) Ibn Hawqal, op. cit., p. 125.

(8) Ibn Hawqal, op. cit., p. 117.

(9) Ibn Hawqal, op. cit., p. 117

(10) Ibn Hawqal, op. cit., p. 195 
I tributi prelevati ogni anno ai bizantini della Calabria consistono una parte delle entrate dell'isola siciliana.

\section{DESCRIZIONE DI UNA CITTÀ MUSULMANA: PALERMO}

Ibn Hawqal dedica numerose e preziose righe alla descrizione topografica di Palermo; "la sola città (dell'isola) celebre e reputata è Palermo, capitale della Sicilia. E' situata sul bordo del mare e si compone di cinque quartieri vecini gli uni gli altri» (11).

La sua espanzione è confrontabile a quella di Corfù.

La Sicilia musulmana vede la rinascita di Palermo; come la Spagna, essa reppresenta è abitata da numerosi commercianti, essa è un centro di commercio, e ciò è dimostrato dalla sua pianta e dai numerosi mercati enumerati: "essa è provvista di un mercado che la attraversa dall'est all'ovests e che chiamano la Grande Strada, che pavimentata di ciottoli, è occupata in tutta la sua lunghezza da edifici commerciali» (12).

Ibn Hawqal ci dice che la moschea cattedrale, "vecchia chiesa cristiana" (13). forma una massa impressionante e puó darci un'idea dell'opulenza e della densità della popolazione: "ho infatti stimato, un giorno che essa era piena di gente, ch'essa poteva contenere più di settemila persone» (14).

Palermo offre le stesse forme di crescita di Corfù; dalla conquista della città, questa si è dilatata topograficamente e demograficamente. Al centro si trova la vecchia città, contorniata dai nuovi sobborghi. Una muraglia di pietre, "molto alta e formidabile" (15), a nove porte, nominate con il loro nome e minuziosamente descritte da lbn Hawqal, cinge la parte vecchia. Tutt, intorno si distinguono quattro quartieri:

- Khalisa, la città dei principi ed ammnistrativa. Essa è protetta da un muro a quattro porte che racchiude il palazzo, gli uffici, la guarnigione militare e I'arsenale;

- il quartiere degli Slavi, quartiere che si distingue per l'origine dei suoi abitanti, è più popolato degli altri. Con una larga facciata marittima, protegge il porto commerciale e i suoi marciapiedi. Dei corsi di acqua servono da separazione tra la cecchia Palermo e questo quartiere;

- il quartiere della Moschea di Ibn Saqlab, anch'essa molto grande. Alla

\footnotetext{
(11) Ibn Hawqal, op. cit., p. 117.

(12) Ibn Hawqal, op. cit., p. 121.

(13) Ibn Hawqal, op. cit., p. 117.

(14) Ibn Hawqal, op. cit., p. 119 .

(15) Ibn Hawqal op. cit., p. 117
} 
sua estremità scorre un ruscello largo e rapido sulle cui rive girano dei mulini. Esso contiene degli orti e dei giardini;

- infine il quartiere Nuovo, il cui nome testimonia la recente costruzione dell'agglomerato.

II quartiere degli Ebrei si trova nella vecchia Palermo.

I principali mercati della città e le differenti corporazioni di mestiere sono situati tra la moschea di Ibn Saqlab e il quartiere Nuovo; il geografo le enumera una ad una. Le moschee sono estremamente numerose in Sicilia: "non conosco una tale profusione di moschee in alcum luogo, né in alcuna grande città, persino con superficie doppia (...) più di trecento moschee, la maggior parte in buone condizioni e solide sotto i loro tetti» (16) solamente in Palermo.

L' acqua potabile degli abitanti della città proviere dai ruscelli o dai pozzi che si trovano in ogni dimora, molto apperezzata "perchè i siciliani mangiano molte cipolle" (17).

\section{QUADRO DELL'ECONOMIA SICILIANA}

«Per ciò che riguarda la fertilità del paese e la sua produzione agricola (...) la Sicilia è confrontabile alle contrade di cui noi abbiamo, all'inizio di questo libro, vantato la fecondità e la prosperità nel passato sin dai tempi antichi» (18). Così, secondo Ibn Hawqal, la prosperità della Sicilia non è che un ricordo: quando egli la percorse, cioè a un'epoca che egli defini essere "la più fiorente e la più abbondante» (19), la situazione dell'isola è peggiorata. Gli abitanti sono divenuti dei barbari che negligiano tutte le forme di commercio, che hanno abbandonato tutte le forme di ospitalità nei confronti degli stranieri e dei viaggiatori.

Nel suo insieme il quadro tracciato dall'autore è molto negativo. La fragilità dell'economia siciliana è manifesta, soprattutto perchè l'isola dipende quasi interamente dalle importazioni:

«i loro interessi vitali dipendono dagli importatori, ed essi hanno un urgente bisogno che i commercianti li visitino. In effetti, riportandoci alla lista delle migliori risorse dei differenti paesi, quest'isola non produce che del grano, della lana, delle pelli di animali, del vino, delle quantità insignificanti di zucchero candito e qualche tessuto di linow (20).

(16) Ibn Hawqal, op. cit., p. 119.

(17) Ibn Hawqal, op. cit., p. 122.

(18) Ibn Hawqal, op. cit., p. 129.

(19) Ibn Hawqal, op. cit., p. 129.

(20) Ibn Hawqal, op. cit., p. 130. 
Tutte le altre derrate di prima necessità devono essere importate, "persino quelle di cui si ha più bisognon(21). Non passa un'annata senza che i raccolti di frumento e di cereali si guastino a causa della negligenza dei contadini.

Così a parte le staffe, por le quali il commerciante lbn Hawqal manifesta un interesante particolare, in Sicilia non cè niente di buono, né di apprezzabile:

"Ma bisogna rendere giustizia alle loro stoffe, poichè esse sono di una qualità e di un prezzo incomparabile. La loro fabbrica, che produce dei tessuti in due forme ordinarie, vende i suoi capi tra cinquanta e sessanta ruba'i. Esse sono ben superiori alle stoffe analoghe che si possono trovare in Egitto, e che sono messe in vendita tra i cinquanta e i sessanta denari» (22).

Ciò nonostante, nei dintorni di Palermo, tutta una zona adibita al giardinaggio serve a nutrire gli abitanti della città; molti corsi d'acqua fanno girare i mulini.

"Lungo questi corsi d'acqua, dalla sorgente alla foce, ci sono lande mercantili coperte di sterpaglia, in cui cresce la canna di Persia, dove si trovano giardini con legumi e produttivi campi di cocomeri. Su questi terreni si raccoglie soprattutto del papiro con cui si fabbricano dei rotoli per scrivere. Questo papiro siciliano è il solo, su tutta la faccia della terra, che possa entrare in rivalità con il papiro egiziano" (23).

Esso è utilizzato soprattutto per i cavi delle ancore delle navi. Solo una piccola parte è riservata alla fabbricazione di rotoli destinati ai documenti ufficiali: "non se ne fabbricano più del bisogno" (24).

L'isola è particolarmente ricca di sorgenti e di ruscelli, e queste acque sono utilizzate per l'irrigazione dei giardini: "la maggior parte dei corsi d' acqua a nord della città sono impiegati per l'irrigamento dei giardini per mezzo di norias» (25). I frutteti sono abbondanti e produttivi, ci sono anche delle vigne destinate alla produzione di vino.

Infine, il paese possiede una mina di ferro, i cui estratti sono impiegati per i bisogni della flotta e delle carrozze del sovrano: «questi giacimenti appartenevano agli Ahghlabita e forniva loro importanti entrate" (26).

(21) Ibn Hawqal, op. cit., p. 130.

(22) Ibn Hawqal, op. cit., p. 130.

(23) Ibn Hawqal, op. cit., p. 121.

(24) Ibn Hawqal, op. cit., p. 121.

(25) Ibn Hawqal, op. cit., p. 122.

(26) Ibn Hawqal, op. cit., p. 122. 


\section{GIUDIZI ESPRESSI SULLA POPOLAZIONE DELL'ISOLA}

Il profondo disprezzo e l'esplosione di antipatie manifeste dal nostro geografo per la società e la cultura siciliana, danno a tutto il capitolo consacrato alla Sicilia un'andatura diffamatoria; il tono è francamente ostile.

Nonostante ciò, quando Ibn Hawqal mette piede in Sicilia alla fine del X secolo, l'isola conosce un periodo assai prospero. E'veroche, la Sicilia è a quest' epoca una regione da poco islamizzata e possiede un livello di civilizzazione meno sviluppato del resto del mondo musulmano.

Noteremo che i giudizi di lbn Hawqal sul poco valore intellettuale e morale degli arabi di Sicilia, dovuto secondo lui all'abuso che questa gente fà della cipolla, restano molto sommari; «la conseguenza dell'abitudine di mangiare cipolle, è che in questa città, non si trovano più persone intelligenti, né abili, né competenti in un ramo qualsiasi delle scienze, né animati da sentimenti nobili e religiosi; anche la maggior parte della popolazione ha dei bassi istinti. Per la maggior parte sono della gente vile e senza valore, senza intedimento e senza una pietà reale. Sono per la maggior parte dei Barqajana e dei libertini che si aggrappano a un popolo che ha conquisto il paese ed è morton (27).

La denigrazione dei siciliani si fa ancora più pronunciata per i maestri di scuola. E'per loro che la malizia si fa più virulenta; uomini disprezzabili che si refugiano nel upiù vile degli impeghi, il più umile dei mestieri, l'occupazione più abietta» per sostrarsi a "un'obbligazione imposta da Dio: la guerra santa, con i suoi onori e le sue glorien (28).

"I maestri di scuola sono abbondanti in quest'isola poiché ci sono scuole inogni località. Sono di differenti categorie, ed occupano differenti gradi nel campo dello squilibrio e dell'imbecillità, in tutti i modi superando la demenza dei maestri di tutti i paesi e degli imbecilli di ogni regione. Questo va tanto Iontano che essi discutono sul sovrano, sul suo modo di vivere, le sue preferenze, ed essi impiegano espressioni ingiuriose parlando dei suoi difetti imputando persino le sue buone qualità alle sue traverse (29).

Ibn Hawqal ha consacrato tutt'un'opera alla Sicilia, libro in cui ha largamente amplifiato il suo giudizio sugli abitanti, «insistendo sulla loro povera intelligenza, la facilità delle loro stolterie, cosí come la loro costante negligenza, la loro scandalosa ammirazione mutuale, i loro scandali, l'insipidità del loro nutrimento, che accresce ancor più la loro stupidità e la loro vergognosa abitudine di credere solamente in se stessim (30).

(27) Ibn Hawqal, op. cit., p. 123.

(28) Ibn Hawqal, op. cit., p. 125.

(29) Ibn Hawqal, op. cit., p. 125.

(30) Ibn Hawqal, op. cit., p. 128. 
Il giudizio sfavorevole si estende anche agli abitanti dei conventi militari; Ibn Hawqal ci dice che sono i luoghi di incontro dei cattivi soggetti del paese che trovano cosí modo di vivere al di fuori della società regolare, alle spese della gente devota e caritatevole.

Di fronte a un tono tanto ostile e ad una mancanza di oggettività tale, un'attitudine di prudenza è di rigore. Non dimentichiamo che il nostro viaggiatore è un orientale puro, un Arabo originario della alta Mesopotamia; in Sicilia deve esser rimasto scioccato del miscuglio etnico della popolazione, e per il livello di civilizzazione sensibilmente meno sviluppato. In più egli è nettamente scandalizzato per la mancanza di pratiche religiose e la corruzione dei sentimenti religiosi dei siciliani, i quali osano contrattare dei matrimoni con i cristiani:

"La maggior parte degli abitanti delle piazze forti, delle zone rurali e dei villaggi sostengono l'opinione che è permesso concludere dei matrimoni condonne cristiane a condizione che i ragazzi seguano unioni restano cristiane come la madre" (31).

Essi trascurano tutti i doveri imposti dall'Islam:

«Essi non fanno le preghiere, non si purificano, non danno l'elemosina legale, non fanno pellegrinaggi; ci sono coloro che osservano il digiuno del ramadan e che mentre digiunano, compiono la grande abluzione dopo un'impuritàn (32).

Infine il capitolo termina con la descrizione delle sórdide abitazioni della popolazione locale.

La Sicilia è dunque una regione in cui la specificità dell'Islam si diluisce e si dimentica. Non sembra ci siano motivi politici che giustificano il tono di queste pagine consacrate agli Arabi di Sicilia. I sentimenti pro-fatimidi già rilevati in Ibn Hawqal, non possono quí giocare un ruolo che sul piano religioso, poiché la Sicilia, politicamente si trova sotto la dominazione dei Fatimidi.

Nonos ciò possiamo rilevare qualche allusione sull'indocilità dei Siciliani, rispettivamente le ribellioni causate nei conventi militare per sottrarsi all'autorità del principe, e la mancanza di entusiasmo per compiere il dovere della guerra santa, difetto che agli occhi del żelante musulmano è decisamente imperdonabile.

Un elemento che ci deve incitare ad avere serie riserve ssulle informazioni date in queste poche pagine, è che lbn Hawqal a Palermo non ha frequentato i letterati; egli ha avuto contatti solamente con dei mercanti non instruiti, e con umili maestri di scuola così numerosi sull'isola. Non dimentichiamo in-

(31) Ibn Hawqal, op. cit., p. 128.

(32) Ibn Hawqal, op. cit., p. 128. 
fatti che l'autore à un uomo colto, formato dall' "adab'; quando esprime un giudizio, egli si riferisce obbligatoriamente a un modo di rapporti interni basati sull'onore, l'etichetta, la tavola e la conversazione. II nostro geografo non ci dice niente sulla dinastia regnante e degli altri dignitari di stato; egli non ha incontrato rappresentanti della classe colta, che dovevano di certo non mancare in una città come Palermo.

In tal modo, i giudizi così severi portati dall'autore sulla Sicilia kalbita manifesta una particolarità evidente e totale; miserie materiale, intellettuale e morale, vanità, ipocrisia, xenofobia, grossolanità, eterodossia, caratterizzano secondo lui la popolazione di quest'isola.

L'atteggiamento negativo preso da Ibn Hawqal, può spiegarsi nella seguente maniera: confrontato al centro iracheno, all'Egitto fatimida o alla Spagnia omeyada, di cui il nostro viaggiatore ha potuto visitare le grandi metropoli ed ammirare l'alta civilizzazione, la Sicilia islamizzata de appena un secolo doveva sembrargli forzatamente di un livello culturale e sociale inferiore. Nei confronti del resto del mondo islamico, la regione doveva avere ai suoi occhi un incontestabile carattere proviciale.

D'altra parte, il miscuglio di popolazioni e i rapporti stabiliti con i cristiani, ciò che deplora il geografo, hanno ai miei occhi tutt'altro valore; per noi significa che la Sicilia sin da questa epoca fu una terra di tolleranza e questa vocazione si confermerà nei secoli seguenti.

\section{Edizioni e traduzioni di IBN HAWQAL}

M. AMARI ha pubblicato il testo arabo e la traduzione francese del capitolo sulla Sicilia nel Journal Asiatique, $V, 1845$, pp. 73-114; dello stesso capitolo una sua traduzione italiana à in Archivio Storico /taliano, serie IV, Firenze, 1847, appendice n. 16; cfr, pure la sua notissima Biblioteca arabo-sicula, resto arabo, Lipsia 1857, pp. 4-11; trad. italiana (in due volumi) Torino e Roma, 1880-81, I vol., pp. 10-27.

IBN HAWQAL, Surrat al-ard, testo arabo edito a cura di M. J. De Goeje in Bibliotheca Geographorum Arabicorum II, Lugd. Bat., 1873

Opus geographicum auctore Ibn Hawkal sec. textum et imagines codicis constantinopolitani... ed. J. H. Kramers, Lugd. Bat., 1938-39.

IBN HAWOAL, Surat al-ard" (ristampa della edizione Kramers), Beirut, 1963.

IBN HAWOAL, Configuration de la terre, trad. francese a cura di J. H. Kramers e G. Wiet, París-Beyrouth, 1964 , in 2 volumi.

- Se si fa a meno dei segni diacritici: Sûrat al-ard. 